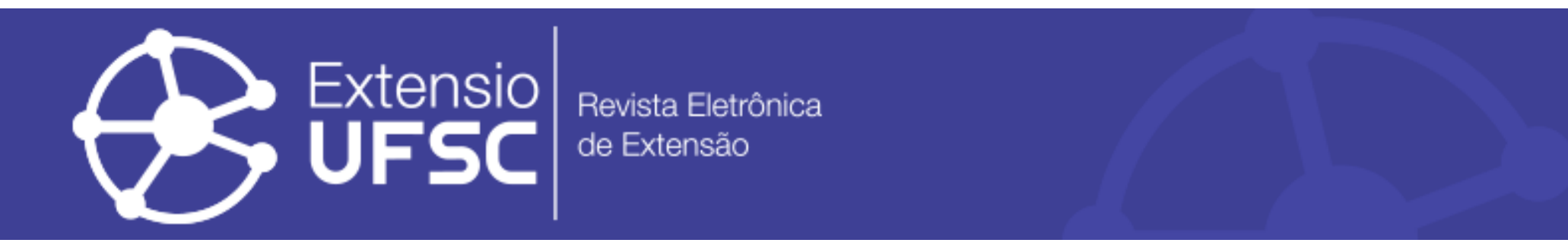

\title{
CONTRIBUIÇÕES DA GINÁSTICA LABORAL PARA QUALIDADE DE VIDA DOS SERVIDORES DE UMA UNIVERSIDADE ESTADUAL
}

\author{
Ana Gabriela Alves Medeiros \\ Universidade do Estado da Bahia \\ gabimedeirosef@gmail.com \\ Ramon de Souza Tomaz \\ Universidade do Estado da Bahia \\ tomazramongbi@hotmail.com
}

\author{
Karina Silva Carvalho \\ Universidade do Estado da Bahia \\ karinacarvalho1201@gmail.com \\ Saul Ricardo dos Santos \\ Universidade do Estado da Bahia \\ saul.cis7@hotmail.com \\ Ingred Laila da Silva Almeida \\ Universidade do Estado da Bahia \\ ingredsilva@live.com
}

\begin{abstract}
Resumo
Este trabalho trata-se de um relato de experiência sobre um projeto de extensão desenvolvido na Universidade do Estado da Bahia - Campus XII (Guanambi), que teve como objetivo oferecer aulas de ginástica para os trabalhadores dessa instituição, a fim de contribuir para a qualidade de vida dos mesmos, sobretudo, para sensibilizá-los para adoção de um estilo de vida saudável. As aulas ocorreram entre abril e novembro de 2017. Este relato é oriundo das observações registradas durante as aulas e do questionário aplicado aos participantes ao final do projeto. Foi possível identificar as contribuições da Ginástica Laboral para os servidores através das expressões ao realizarem as atividades, em especial nas recreativas, nas expectativas quanto às atividades seguintes, na interação social atuando sobre a timidez de alguns participantes, nos diálogos informais a respeito da permanência do projeto, nas respostas dos questionários e, no tocante, a diminuição de dores musculares, e na compreensão da importância da Ginástica Laboral.
\end{abstract}

Palavras-chave: Trabalhador. Bem Estar. Exercício Físico.

\section{CONTRIBUTIONS OF THE LABORAL GYMNASTICS TO THE QUALITY OF LIFE OF THE EMPLOYEES OF A STATE UNIVERSITY}

\begin{abstract}
This paper is an experience report about an extension project developed at Universidade do Estado da Bahia - Campus XII (Guanambi). The project aims to offer workplace exercises for the employees of this institution, in order to contribute to their quality of life, and to sensitize them to adopting a healthy lifestyle. The classes took place between April and November of 2017. This report comes from the observations during the classes and the questionnaire applied to the participants at the end of project. It was possible to identify the contributions of the laboral gymnastics to the servants through the manifestations when they did the activities, especially the recreational ones, also through their social interactions, conversations about the continuity of the project, their answers to the questionnaires, mainly regarding the decrease of muscular pains, and finally, their understanding about the importance of the workplace exercises.
\end{abstract}

Keywords: Workers. Welfare. Physical Exercises.

\section{CONTRIBUCIONES DE LA GIMNASIA LABORAL PARA CALIDAD DE VIDA DE LOS SERVIDORES DE UNA UNIVERSIDAD ESTATAL}

\section{Resumen}

Este artículo se trata de un relato de experiencia sobre un proyecto de extensión desarrollado en la Universidade do Estado da Bahia - Campus XII (Guanambi), que tuvo como objetivo ofrecer clases de gimnasia para los trabajadores de dicha institución, a fin de contribuir a la calidad de vida de los mismos y sensibilizarlos para la adopción de un estilo de vida saludable. Las clases ocurrieron entre abril y noviembre de 2017. Este relato es oriundo de las observaciones durante las clases y del cuestionario aplicado a los participantes al final del proyecto. Fue posible identificar las contribuciones de la Gimnasia Laboral a los servidores a través de las expresiones al realizar las actividades, en especial en las recreativas, en las expectativas en cuanto a las actividades siguientes, en la interacción social entre los participantes, en los diálogos informales acerca de la permanencia en el proyecto, los relatos sobre la disminución de los dolores musculares, y la comprensión de la importancia de la Gimnasia Laboral.

Palabras clave: Trabajador. Bienestar. Ejercicio Físico. 
Contribuições da ginástica laboral para qualidade de vida dos servidores de uma universidade estadual

\section{CONTEXTUALIZAÇÃO}

Devido à globalização, foi instaurado um novo modelo de produção, provocando uma intensificação organizacional, e consequentemente o aumento expressivo à carga horária e rendimento da classe trabalhadora. Esse novo parâmetro solicita elevados níveis de concentração e entre outras exigências que repercute de forma negativa na saúde física e psíquica de seus trabalhadores, visto que quando o indivíduo se encontra em situações constantes de pressão ocupacional, lhe-é gerado um desequilíbrio corporal provocando reações adaptativas comportamentais, afetivas, cognitivas e fisiológicas denominado de estresse ocupacional (ANDRADE et al., 2015).

Nesta perspectiva, é cabível ressaltar o quanto os aspectos físico-motores são afetados em determinadas condições como as longas jornadas de trabalho repletas de movimentos monótonos e repetitivos, que se tornam influentes ao surgimento de Lesões por Esforço Repetitivo (LER), Distúrbios Osteomusculares Relacionado ao Trabalho (DORT), lombalgia, dentre outras doenças (ANDRADE et al., 2015).

Embasados nesse contexto, entre os anos de 1980 e 1990, a Ginástica Laboral (GL) chegou ao Brasil, objetivando a prevenção de doenças ocupacionais, acidentes no trabalho, aumento da produtividade e melhora no bem estar dos trabalhadores. Atualmente a GL é compreendida como o conjunto de atitudes com o intento no enfrentamento dos distúrbios físicos e emocionais tratando-se da saúde do trabalhador (SOARES, ASSUNÇÃO e LIMA, 2006). Para além da prevenção de lesões e auxilio na reabilitação, a GL ainda propicia a melhoria geral do funcionamento do aparelho músculo-esquelético, contribuindo de forma expressiva em longas situações de movimentos posturais isométricos.

A GL apresenta-se ainda mais eficaz quando definida em comum acordo com a atividade desempenhada pelo trabalhador e suas necessidades. Haja vista que quando desenvolvida no início do expediente apresenta-se com características de aquecimento músculo-esquelético e articular, objetivando o alcance dos músculos e articulações que serão utilizados no decorrer do expediente a fim de poupá-los de acidentes como as distensões (POLITO e BERGAMASCHI, 2002).

Quando desenvolvida no meio do expediente, a GL se apresenta com características de prevenção do estresse do ambiente, assim como das tensões musculares e da fadiga. E quando realizada ao término do expediente se apresenta com características de relaxamento, objetivando externar as tensões adquiridas no decorrer do trabalho (SOARES, ASSUNÇÃO e LIMA, 2006). 
Contribuições da ginástica laboral para qualidade de vida dos servidores de uma universidade estadual

Decerto que o caráter assumido pela prática da GL também se relaciona com os objetivos estabelecidos e com os exercícios desenvolvidos.

De todo modo, a GL possibilita o desenvolvimento de valências físicas, tais como força, flexibilidade, agilidade e coordenação motora. Assim, pode proporcionar aos praticantes uma maior disposição física, satisfação ao realizar as atividades diárias, melhoria no desempenho laboral e nas relações interpessoais.

Segundo Silva (2007), quando falamos de saúde estamos nos remetendo ao estado multidimensional que envolve aspectos fisiológicos, afetivos, motores e sociais, não limitando a mesma a ausência de doenças. Nesse sentido, podemos considerar a prática regular de atividade física como um importante fator que interfere diretamente de forma positiva na manutenção da saúde.

Neste âmbito, a GL pode proporcionar tanto para o trabalhador quanto para a empresa ou instituição a qual está vinculada resultados diretos relacionados às relações interpessoais no ambiente de trabalho, ao alívio ou diminuição de dores e desconfortos corporais e ao desenvolvimento das tarefas laborais. A universidade, de certo modo, funciona como uma empresa, sendo dividida em diversos setores para atender a demanda dos serviços que são oferecidos pela mesma. Assim, à disposição física e mental de seus funcionários é fundamental para a eficiência do trabalho ofertado.

Este trabalho relata a experiência de um projeto de extensão denominado Ginástica Laboral para servidores da Universidade do Estado da Bahia - Campus XII. Tal projeto teve como objetivo oferecer atividades de Ginástica Laboral para os trabalhadores da referida instituição, a fim de contribuir para a qualidade de vida dos mesmos, sobretudo, no que se refere à orientação e oportunidade de prática regular de exercícios físicos e também para a adoção de um estilo de vida saudável dentro e fora do ambiente de trabalho.

\section{METODOLOGIA}

O projeto foi desenvolvido no período de 04 de abril a 30 de novembro de 2017, e contou com a participação de 18 funcionários (5 homens e 13 mulheres) de diversos setores do campus, com faixa etária entre 23 e 58 anos. Os servidores estiveram divididos em três grupos de acordo com suas disponibilidades. Eram realizadas três aulas por semana com duração média de 20 minutos. Estas aulas eram ministradas por dois discentes do curso de Educação Física da referida universidade, os quais eram bolsistas do projeto de extensão, e eram supervisionados, orientados e avaliados pela professora idealizadora do projeto. 
Contribuições da ginástica laboral para qualidade de vida dos servidores de uma universidade estadual

As atividades foram elaboradas com a intenção de proporcionar aos praticantes movimentos corporais que eles não realizavam com frequência em seu ambiente de trabalho. As aulas eram direcionadas para a melhoria de valências físicas como força, flexibilidade, equilíbrio e coordenação, estabelecendo níveis de complexidade gradativos diante da desenvoltura dos praticantes. Além disso, eram realizadas atividades recreativas com o intuito de desenvolver a sociabilidade e integração entre os participantes.

Inicialmente, foi efetuada uma avaliação diagnóstica a respeito do estilo de vida dos funcionários através de um questionário, no qual continha questões fechadas acerca da prática de atividade física, hábitos alimentares, desconfortos e estresse no trabalho. Todas as informações coletadas serviram como base para a estruturação das atividades relacionadas à Ginástica Laboral.

No decorrer do processo utilizamos a observação direta e conversas com os participantes para analisar e avaliar o desenvolvimento do projeto, bem como a percepção dos praticantes perante as atividades da GL.

Ao final do projeto, utilizamos novamente um questionário como ferramenta de avaliação das aulas do projeto, e das contribuições do mesmo para a qualidade de vida dos servidores. $\mathrm{O}$ questionário continha nove perguntas objetivas acerca das aulas de ginástica laboral, sobretudo relacionadas à participação, motivação, satisfação e percepção dos participantes, e uma questão aberta para comentários dos servidores.

\section{PERCEPÇÃO DOS SERVIDORES ACERCA DAS AULAS DE GINÁSTICA LABORAL}

Ao longo do processo de intervenção e com a maior aproximação dos grupos, ficou claro o comprometimento de alguns participantes, que sempre se apresentavam dispostos a colaborar com as atividades e relatavam constantemente o quanto a prática dos exercícios ajudava a descontrair e diminuir o estresse, além de servir como uma válvula de escape aos longos períodos sentados ou realizando a mesma tarefa.

Em contrapartida, em um dos grupos, a evasão apresentou-se de forma significativa, o que dificultou o desenvolvimento das atividades propostas. Através das conversas com os participantes pode-se perceber que a falta de interesse em realizar a GL encontrava-se atrelada a fatores como o não entendimento da relevância da atividade, e, sobretudo, ao fato de caracterizarem a GL como uma prática que não influenciava diretamente os fatores estéticos.

Segundo Martins (2008), existe uma resistência do trabalhador em relação à mudança em sua rotina de trabalho, que afeta diretamente a mudança de hábitos, comportamento o qual interfere no processo de adesão do mesmo ao programa. Estudos também evidenciam que os 
Contribuições da ginástica laboral para qualidade de vida dos servidores de uma universidade estadual

trabalhadores com sobrecarga manual preferem utilizar seu tempo de pausa para sua recuperação física e psíquica (SOARES, ASSUNÇÃO e LIMA, 2006). Assim, embora as atividades fossem adequadas às funções desempenhadas pelos servidores, compreende-se a não continuidade de alguns trabalhadores no projeto.

Outro aspecto relevante percebido durante o desenvolvimento das aulas diz respeito a predileção a um determinado tipo de atividade em detrimento de outras, em que as aulas com cunho recreativo obtinham destaque, visto que a participação nestas aulas, em todos os grupos, era maior do que nos outros dias, além da maior interação e dinamicidade entre os participantes. Por esse motivo, ao longo do processo, as aulas foram reformuladas, visto que as duas primeiras aulas da semana eram destinadas ao desenvolvimento das valências físicas e durante a última aula eram acrescidas as atividades recreativas. Deste modo, foram acrescentadas atividades lúdicas e com raciocínio lógico durante todas as aulas da semana, o que gerou gradativamente uma maior participação.

O incentivo dos companheiros de trabalho também se apresentou como parte primordial para o processo, visto que a partir do momento que os participantes percebiam o ambiente de descontração e estímulo do colega, se sentiam mais dispostos e interessados a participar, pois não havia pressão diante do erro, por esse motivo a coletividade tornou-se uma peça de fundamental importância.

Quando pensado em relação aos benefícios da GL para uma melhor relação interpessoal leva-se em consideração que os seres humanos têm em sua essência a necessidade de se relacionar. A todo momento, nos diversos ambientes, os indivíduos convivem uns com os outros, formam-se grupos por afinidade e aproximações que perpassam por vezes a religião, laços familiares e convívio de trabalho. E através dessa socialização estabelecem as suas capacidades afetivas e de cooperação.

De acordo com Silva e colaboradores (2007), os pontos positivos advindos da interação interpessoal referem-se à liderança democrática, diálogo, respeito, responsabilidade e bom humor. Nesse mesmo sentido, Leitão, Fortunato e Freitas (2006) discorrem que as experiências propiciadoras do sentimento afetivo orientam o domínio de conduta e aceitação do próximo no que diz respeito ao convívio interacional, ressaltando que tanto as emoções positivas quanto as negativas inferem no ambiente de trabalho.

Diante disso, a partir das observações e relatos dos funcionários, identificou-se um ambiente de trabalho significativamente mais interativo por meio do estabelecimento de laços positivos de amizade e cooperação desenvolvidos nas aulas de Ginástica Laboral. 
Contribuições da ginástica laboral para qualidade de vida dos servidores de uma universidade estadual

\section{PERCEPÇÃO DOS SERVIDORES ACERCA DAS CONTRIBUIÇÕES DA GINÁSTICA LABORAL}

A partir das informações obtidas no início do projeto, e a partir do questionário, podemos constatar o baixo nível de atividade física entre os participantes, fato que pode ser atribuído a diversos fatores, tais como as comodidades da vida moderna, pois a maioria dos participantes indicou que utilizavam carro ou moto para se locomover ao trabalho. Além disso, alegaram também que passavam boa parte do tempo sentados devido a sua função, e em virtude de sua carga horária de trabalho acusavam não ter tempo para realizar exercícios físicos.

Tais dados convergem com os constatados em uma pesquisa realizada por Silva e Juvêncio (2004), que analisou os Fatores Humanos Gerais (FHG) e o nível de Aptidão Física Relacionada à Saúde (AFRS) em servidores da Universidade Federal de Viçosa (Minas Gerais). Os pesquisadores identificaram que as atividades desenvolvidas pelos funcionários, bem como o estilo de vida tem potencializado fatores negativos para a saúde dos mesmos.

Neste sentido, a promoção da prática de exercícios físicos no local de trabalho para os servidores da UNEB caracterizou-se como uma iniciativa de educação para a saúde, cujos exercícios eram acompanhados de explicações sobre seu objetivo, sua relevância e possibilidades de variações. Desta forma, as aulas buscavam conduzir os servidores a descoberta dos benefícios da prática de exercícios, favorecendo a vivência de atitudes positivas em relação à saúde, subsidiada pelos conhecimentos adquiridos, bem como pelas conquistas físicas e sociais, motivando os mesmos para a realização de exercícios físicos para além da ginástica laboral.

Os exercícios realizados objetivavam melhorar a aptidão física relacionada à saúde, bem como a qualidade de vida dos servidores. Contudo, para o usufruto destes objetivos era necessário que os funcionários realizassem a prática da ginástica com regularidade, além de aderirem às mudanças em seus estilos de vida.

No início do projeto, os funcionários alegaram a incidência de dores na coluna, ombros e punhos. No entanto, inicialmente os participantes encontravam dificuldade para relacionar a prática regular da GL como um subsídio, que a longo prazo, interviria positivamente nestes desconfortos. Sabe-se que as tensões musculares secundárias e o estresse possuem uma estreita relação com as variáveis psicossociais e a biomecânica humana. Diante desse quadro, a adoção da GL como parte de um programa de promoção de saúde do trabalhador pode proporcionar uma diminuição dos casos de distúrbios osteomusculares, bem como o alívio das dores corporais (SAMPAIO e OLIVEIRA, 2008). Tal fato foi constado ao final do projeto, a partir das falas dos participantes. Foi também perceptível no decorrer do projeto a evolução dos servidores ao 
Contribuições da ginástica laboral para qualidade de vida dos servidores de uma universidade estadual

realizarem as atividades com mais destreza diante do aumento do nível de complexidade dos exercícios.

Ademais, os resultados apresentados no questionário aplicado após o projeto indicaram que $83 \%$ dos participantes consideraram as aulas de Ginástica Laboral como um componente motivador para a realização de exercícios físicos fora do ambiente de trabalho. Assim, reconhecemos que o objetivo do projeto de sensibilizar os servidores sobre a importância da prática de exercícios físicos regulares, sobretudo, para a adoção de um estilo de vida saudável dentro e fora do ambiente de trabalho foi relativamente alcançado.

Diversas pesquisas indicam a relevância do programa de ginástica laboral para a melhoria da qualidade de vida de seus participantes, assim como a aderência e manutenção de práticas de exercício físico fora do ambiente de trabalho (OLIVEIRA, 2006; POLITO, BERGAMACHI, 2002; MILITÃO, 2001; dentre outros). Sobretudo, é necessário que estas aulas sejam adequadas, organizadas e orientadas para as especificidades dos trabalhadores.

Com as informações obtidas em cada etapa do projeto foi possível perceber as contribuições da ginástica laboral na promoção da melhoria na qualidade de vida dos trabalhadores, de maneira que os funcionários se consideraram satisfeitos com essa iniciativa e com os impactos do projeto em suas habilidades físicas, cognitivas e sociais.

Numa perspectiva geral, ao analisar as respostas dos participantes, podemos alegar que as pessoas envolvidas notaram mudanças positivas em seu comportamento relacionado ao estilo de vida, em virtude da prática da ginástica laboral. Todos os respondentes afirmaram que perceberam melhorias nas suas capacidades físicas, diminuição dos desconfortos musculares, bem como melhorias em suas relações de trabalho com a participação nas aulas de ginástica laboral. Os mesmos reconheceram a importância do projeto e sua permanência, bem como deram sugestões como o aumento na quantidade de aulas durante a semana e mais atividades recreativas.

\section{CONSIDERAÇÕES FINAIS}

Desde o início até o final do projeto foi possível identificar as contribuições da Ginástica Laboral para os servidores, através das expressões faciais e corporais ao realizarem as atividades, em especial nas recreativas, nas expectativas quanto às atividades seguintes, na interação social atuando sobre a timidez de alguns participantes, nos diálogos informais a respeito da permanência do projeto, nas respostas dos questionários quanto aos benefícios físicos e no tocante a diminuição de dores musculares, e na compreensão da importância da GL. 
Contribuições da ginástica laboral para qualidade de vida dos servidores de uma universidade estadual

As estratégias utilizadas, bem como os exercícios físicos aeróbicos, localizados, de relaxamento e recreação realizados nas aulas potencializaram a sensibilização dos servidores da UNEB-Campus XII sobre a importância da prática de exercícios físicos regulares e a promoção do desenvolvimento de aspectos físicos, cognitivos e psicomotores. Sendo assim, compreendemos que as aulas de Ginástica Laboral conduziram os participantes para uma prática e prazer do corpo em movimento.

Acrescenta-se, sobretudo, a melhoria das relações interpessoais entre os servidores que participaram do projeto, o que conflui em benefícios tanto para os sujeitos quanto para a instituição, visto que isto torna o trabalho mais fluido e prazeroso.

Embora não tenha sido o enfoque deste trabalho, devemos destacar também os aspectos inerentes às relações pedagógicas no campo da Educação Física que foram proporcionados aos discentes monitores do projeto, especialmente, no trato pedagógico com o conteúdo da ginástica laboral e no processo de ensino e aprendizagem com os servidores. A extensão constitui-se como um elemento capaz de operacionalizar a relação entre teoria e prática, ampliando o espaço da sala de aula, bem como, um elemento enriquecedor para a formação profissional dos discentes, sendo estes aspectos perceptíveis a partir das reuniões de planejamento e estudos acerca da temática trabalhada.

\section{REFERÊNCIAS}

ANDRADE, Andréa Mattos; et al. Ginástica Laboral: efeitos de um programa de ginástica laboral sobre a flexibilidade em trabalhadores de diversos setores de um hospital de ensino da região do Vale do Rio Pardo/RS. Rev. Cinergis, v. 16, n. 3, p. 209/213, julho/setembro, 2015.

LEITÃO, Sergio Proença; FORTUNATO, Graziela; FREITAS, Angilberto Sabino. Relacionamentos interpessoais e emoções nas organizações: uma visão biológica. Revista de Administração Pública, v, 40, n. 5, p. 883/907, setembro/outubro, 2006.

MARTINS, Caroline de Oliveira. Programa de Promoção de Saúde do Trabalhador-PPST. Jundiaí: Fontoura, 2008.

MILITÃO, Angeliete Garcez. A influência da ginástica laboral para a saúde dos trabalhadores e sua relação com os profissionais que a orientam. 2001. Dissertação (Mestrado em Engenharia de Produção) — Programa de Pós-Graduação em Engenharia de Produção, Universidade Federal de Santa Catarina, Florianópolis.

OLIVEIRA, João Ricardo Gabriel de. A prática da ginástica laboral. 3. ed. Rio de Janeiro: Sprint, 2006.

POLITO, Eliane; BERGAMASCHI, Elaine Cristina. Ginástica Laboral: teoria e prática. $1^{\mathrm{a}}$ Edição. Rio de Janeiro: Sprint, 2002. 
Contribuições da ginástica laboral para qualidade de vida dos servidores de uma universidade estadual

SAMPAIO Adelar Aparecido; OLIVEIRA, João Ricardo Gabriel de. A ginástica laboral na promoção da saúde e melhoria da qualidade de vida no trabalho. Caderno de Educação Física, v. 7, n. 13 , p. $71 / 79,2008$

SILVA A. Antônio J.; MIRANDA NETO Jaime T.; MONTEIRO. Maria V.; REIS, Victor M. Medidas e avaliação. Shape, Rio de. Janeiro; 2007.

SILVA, Cristiano Diniz da; JUVÊNCIO, José de Fátima. Diagnóstico da aptidão física relacionada à saúde em trabalhadores de escritório da Universidade Federal de Viçosa. Revista brasileira de cineantropometria e desempenho humano, 6(1), 63-71, 2004.

SILVA, Danielly Magalhães da; et al. Relacionamento interpessoal no contexto organizacional. In: V Congresso Virtual Brasileiro de Administração - CONVIBRA, 7, 2007. Anais (on-line). Disponível: http://www.convibra.com.br/2008/artigos/289 0.pdf Acesso em: $21 / 06 / 2017$.

SOARES, Raquel Guimarães; ASSUNÇÃO, Ada Ávila; LIMA, Francisco de Paula Antunes. A baixa adesão ao programa de ginástica laboral: buscando elementos do trabalho para entender o problema. Revista Brasileira de Saúde Ocupacional, São Paulo, v. 31, n. 114, p. 149-160, 2006.

Recebido em: 24/08/2018

Aceito em: 27/03/2019 